\title{
Sullivan Awarded Balzan Prize
}

DenNis Sullivan of the State University of New York at Stony Brook and the City University of New York has been awarded the International Balzan Prize for his "major contributions to topology and the theory of dynamical systems, opening new perspectives for generations to come" and for his "exceptional results in many fields of mathematics, such as topology, geometry, the theory of Kleinian groups, analysis and number theory." The Balzan Prizes are awarded to scholars, artists, and scientists who have distinguished themselves in their fields on an international level. The cash prize is 750,000 Swiss francs (approximately US\$800,000). Sullivan was presented with the prize by the president of the Italian Republic during a ceremony held in Rome, Italy, on November 20, 2014.

\section{The Work of Dennis Sullivan}

The Balzan Foundation provided the following information about the work of Dennis Sullivan.

Algebraic topology is the study of topology using algebraic tools. The theory is particularly useful when algebra allows a complete description of topology.

At the beginning of his career, Dennis Sullivan was one of the main proponents of (mathematical!) surgery theory. He made a fundamental contribution to the Hauptvermutung, which concerns the different ways of triangulating space. He also obtained a complete classification of simply connected high-dimensional manifolds, for which the homotopy type is known. His article "Genetics of homotopy theory and the Adams conjecture" represents a remarkable step forward.

Later, he developed (along with Quillen) rational homotopy theory, which is one of the mathematical gems of the twentieth century. A purely algebraic structure-Sullivan's minimal model-makes it possible to completely reconstruct the rational homotopy type of a space. Infinitesimal Computations in Topology is one of the most important texts on algebraic topology in the twentieth century, of similar stature to Poincaré's seminal paper "Analysis Situs."

In the second part of his career, Sullivan transformed the theory of dynamical systems. The study of complex dynamics, initiated by Fatou and Julia at the beginning of the twentieth century, had been neglected until Sullivan applied and developed useful, "quasiconformal" tools from harmonic analysis. The theory was revolutionary. Among his other work on this theme, his "Nonwandering Theorem," with its magnificent proof, is particularly notable.

Sullivan has an overall unitary vision of mathematics. For example, the concept of the Sullivan Dictionary provides parallels for theories that

DOI: http://dx.doi.org/10.1090/noti1198 
Fellow at the Massachusetts Institute of Technology (1969-1973). From 1973 to 1974 he was associate professor at the University of Paris-Orsay and in 1974 was appointed professor at the Institut des Hautes Études Scientifiques (IHES). In 1981 he was appointed to the Einstein Chair at the City University of New York, where he worked jointly with the IHES until 1996. He is currently Albert Einstein Professor of Science and Distinguished Professor of Mathematics at the City University of New York Graduate School and University Center and professor of mathematics at Stony Brook University.

Sullivan was awarded the U.S. National Medal of Science in 2005 and the AMS Steele Prize for Lifetime Achievement in 2006. His other honors include the Oswald Veblen Prize in Geometry (1970), the Elie Cartan Prize in Geometry (1981), the King Faisal International Prize for Science (1994), the Gold Medal of the Brazilian Academy of Sciences (1996), and the Wolf Prize for Mathematics (2010).

He is a member of the U.S. National Academy of Sciences and the New York Academy of Sciences, a fellow of the American Academy of Arts and Sciences, a corresponding member of the Brazilian National Academy of Sciences and the Irish Royal Society, and an honorary member of the London Mathematical Society. He served as vice president of the American Mathematical Society from 1990 to 1993 and was elected a Fellow of the AMS in 2012.

Also receiving the Balzan Prize in 2014 is IAN HACKING, a philosopher who has written about the philosophy of mathematics, including in his latest book, Why Is There Philosophy of Mathematics at All? (Cambridge University Press, 2014).

\section{About the Prize}

The International Eugenio Balzan Prize Foundation was established in 1956 by Lina Balzan, who had come into a considerable inheritance on the death of her father, Eugenio, and began the foundation to honor his memory. The aim of the International Balzan Prize Foundation is to promote culture, the sciences, and the most meritorious initiatives in the cause of humanity, peace, and brotherhood among peoples throughout the world. Currently, four annual awards are made: two in literature, moral sciences, and the arts and two in the physical, mathematical, and natural sciences and medicine. The award fields vary each year and can be related to either a specific or an interdisciplinary field; they look to go beyond the traditional subjects, both in the humanities (literature, moral sciences, and the arts) and in the sciences (medicine and the physical, mathematical, and natural sciences). They give priority to innovative research. Half of the amount received by the winner of each of the four prizes must be destined for research work, preferably involving young scholars and researchers.

- From Balzan Foundation announcements

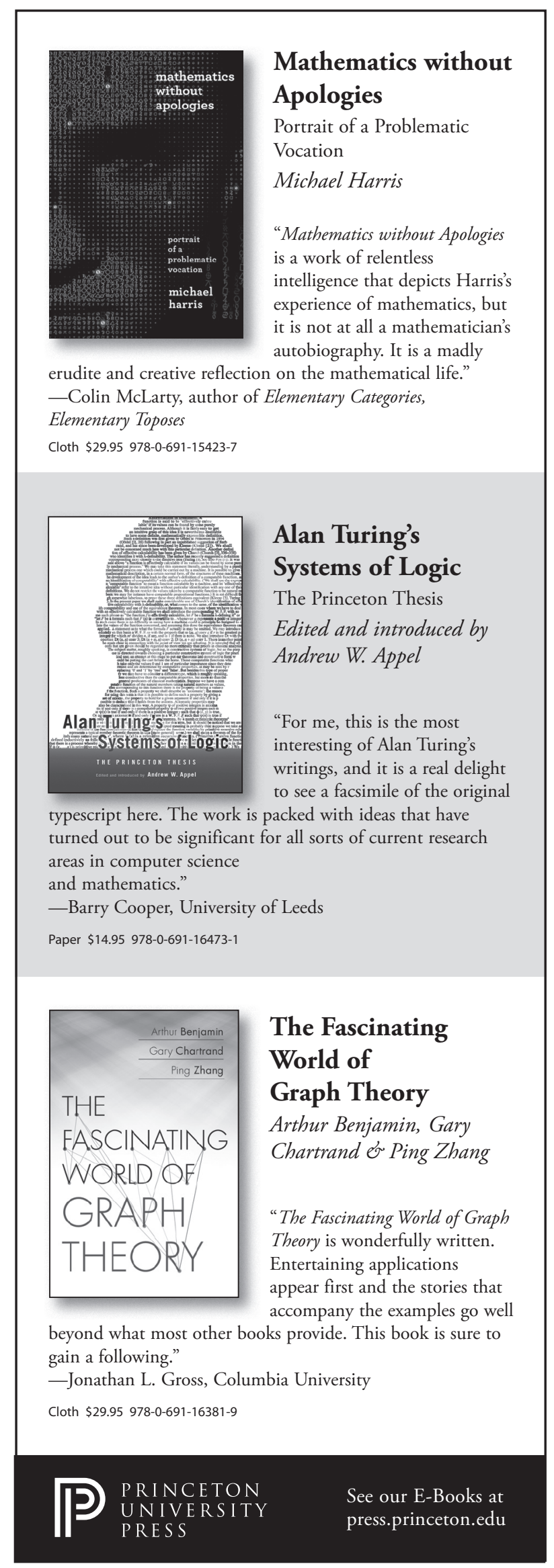

